\title{
La escritura textual narrativa en el aprendizaje de estudiantes en Educación
}

\section{Narrative Textual Writing in the Learning of Students of Education}

\section{Escrita textual narrativa na aprendizagem do aluno em Educação}

\author{
María Luisa Guadalupe Verástica Cháidez \\ Universidad Autónoma de Sinaloa, México \\ maria.verastica@uas.edu.mx \\ https://orcid.org/0000-0002-2306-3181 \\ Crisanto Salazar González \\ Universidad Autónoma de Sinaloa, México \\ crisantosalazar@uas.edu.mx \\ https://orcid.org/0000-0002-8784-346X
}

\section{Resumen}

En este artículo se presentan resultados parciales de una investigación que buscó identificar las prácticas de escritura en alumnos de la licenciatura en Educación Media de la Universidad Autónoma de Sinaloa, con el objetivo de examinar cuáles son los textos en el aprendizaje de la disciplina profesional. La metodología es de corte cualitativo. La recolección de datos fue vía entrevista semiestructurada con enfoque sociocultural. Las entrevistas se aplicaron a 21 sujetos de un grupo de octavo semestre y a cuatro de sus profesores. En los fundamentos teóricos se abordan la práctica social de la cultura escrita, las dificultades al escribir textos académicos, la escritura desde las asignaturas y durante los estudios universitarios, la revisión grupal de discursos que abona a la reescritura personal y la inclusión de centros de escritura. Como parte de los resultados, los alumnos expresaron que destinan en promedio ocho horas por semana para componer y sustentar un escrito; tienen preferencia por la redacción del discurso literario, dificultad para escribir atendiendo la cuestión gramatical, predisposición 


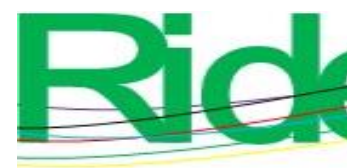

Revista Iberoamericana para la Investigación y el Desarrollo Educativo ISSN $2007-7467$

por trazar una ruta o plan que guiará el texto, una inclinación por redactar en solitario y una tendencia a elaborar textos en un entorno limpio, luminoso, fresco, tranquilo, con mesas grandes, fijas, y con ventanas con vista a árboles. En las respuestas de los profesores destaca que los jóvenes recurren al texto narrativo para darle forma a distintos trabajos solicitados. Dentro de las conclusiones está el hecho de que la composición escritural en la universidad demanda acciones de reescritura en compañía y de forma grupal a fin de promover el aprendizaje personal. Esto apunta a la inclusión de centros de escritura donde sea posible partir de estructuras narrativas para encaminarse hacia la construcción de textos académicos.

Palabras clave: cultura escrita, educación superior, estudiantes, práctica social.

\section{Abstract}

This article presents partial results of an investigation that sought to identify the writing practices in students of the Bachelor of Secondary Education of the Universidad Autónoma de Sinaloa, with the aim of examining which texts are in the learning of the professional discipline. The methodology is qualitative. The data collection was via a semi-structured interview with a sociocultural approach. The interviews were applied to 21 subjects from an eighth semester group and four of their teachers. The theoretical foundations address the social practice of written culture, the difficulties that arise when writing academic texts, writing from subjects and during university studies, the group review of texts that pays for personal rewriting and the inclusion of centers dedicated exclusively to writing. As part of the results, the students expressed that they spend an average of eight hours per week to compose and sustain a writing, there is a preference for writing literary speeches, difficulty in writing considering the grammatical question, predisposition to draw a route or plan before starting to write, an inclination to write alone and a tendency to write texts where it is clean, bright, cool, quiet, with large, fixed tables and windows overlooking the trees. In the teachers' responses, it stands out that young people resort to narrative text to shape different jobs requested. Among the conclusions is the fact that the scriptural composition in the university demands rewriting actions in company and in groups in order to promote personal learning. This points to the inclusion of writing centers where it is possible to start from narrative structures that lead to the construction of academic texts.

Keywords: written culture, higher education, students, social practice. 


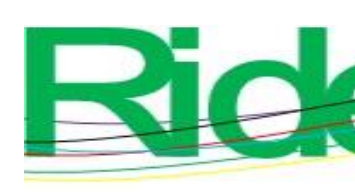

Revista Iberoamericana para la
Investigación y el Desarrollo Educativo
ISSN $2007-7467$

\section{Resumo}

Este artigo apresenta resultados parciais de uma investigação que buscou identificar as práticas de escrita em alunos do Curso de Bacharelado em Educação Secundária da Universidade Autônoma de Sinaloa, com o objetivo de verificar quais textos são utilizados na aprendizagem da disciplina profissional. A metodologia é qualitativa. A coleta de dados se deu por meio de entrevista semiestruturada com abordagem sociocultural. As entrevistas foram aplicadas a 21 sujeitos de uma turma do oitavo semestre e quatro de seus professores. Os fundamentos teóricos abordam a prática social da cultura escrita, as dificuldades na redação de textos acadêmicos, a redação de disciplinas e durante os estudos universitários, a revisão em grupo de discursos que pagam a reescrita pessoal e a inclusão de centros de redação. Como parte dos resultados, os alunos expressaram que gastam em média oito horas semanais para compor e apoiar uma escrita; Eles têm preferência pela escrita do discurso literário, dificuldade em escrever de acordo com a questão gramatical, predisposição para traçar um roteiro ou plano que norteará o texto, inclinação para escrever sozinho e tendência para elaborar textos em ambiente limpo, claro e fresco, tranquilo, com grandes mesas fixas e com janelas que dão para as árvores. Nas respostas dos professores, destaca-se que os jovens recorrem ao texto narrativo para dar forma aos diferentes empregos solicitados. Entre as conclusões está o fato de que a composição das escrituras na universidade exige ações de reescrita em empresa e em grupo, a fim de promover o aprendizado pessoal. Isso aponta para a inclusão de centros de escrita onde é possível partir de estruturas narrativas para avançar na construção de textos acadêmicos.

Palavras-chave: cultura escrita, ensino superior, alunos, prática social.

Fecha Recepción: Octubre 2020

Fecha Aceptación: Marzo 2021

\section{Introducción}

Una problemática que se observa en la Facultad de Ciencias de la Educación (FACE) de la Universidad Autónoma de Sinaloa (UAS) en torno a la cultura escrita es la insuficiencia de prácticas de lectoescritura y educación literaria, puesto que en el programa de la licenciatura en Educación Media (LEM) el espacio curricular asignado al aprendizaje de textos académicos es reducido. En el aula, los estudiantes no cuentan con un tiempo permitido para leer y escribir; en su lugar, se hace discusión teórica de los contenidos. 


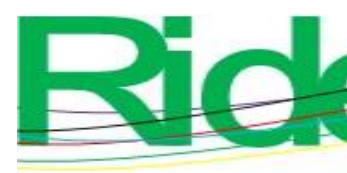

Revista Iberoamericana para la Investigación y el Desarrollo Educativo ISSN 2007 - 7467

Bajo el supuesto de que los estudiantes participan poco en la producción de textos académicos, aun cuando están por concluir sus estudios, se propuso el objetivo de investigar, conocer y analizar en qué medida los aprendices recurrían a actividades escriturales en los últimos cursos de su formación profesional. Si bien estos sujetos fueron seleccionados partiendo del hecho de que sabían escribir discursos académicos, ya que habían llegado al último grado y, por extensión, serían capaces de organizar el tiempo y espacio con antelación, no obstante, dentro de los resultados, a modo de adelanto, $95 \%$ de los estudiantes confesó no escribir en la escuela (el resto sí que lo hacía, redactaba en la escuela, y en solitario). Los estudiantes reportan discursos escritos cuando tienen evaluación y al término del semestre, como productos finales, sin mostrar el proceso de su creación. De ahí que los resultados de esta investigación puedan abonar a retratar el estado actual de las prácticas de escritura en los procesos de formación universitaria.

Para sustentar la indagación, se procedió a revisar las aportaciones teóricas presentadas por investigadores de comunidades científicas, cuyos enfoques van desde el cognitivo hasta el cultural y sociocultural. Entre estas investigaciones, en México, Carrasco, Encinas, Castro y López (2013) plantean lo siguiente:

a) Las prácticas de lectura y escritura varían no solo entre comunidades disciplinarias, también lo hacen entre niveles escolares; $b$ ) que la educación media superior y superior enfrentan al estudiante a nuevas formas de comunicación y le demandan tareas más sofisticadas (p. 349).

De acuerdo con Salazar (2015), "los docentes tienden a desarrollar más la parte lingüística de la producción escrita de los jóvenes, desdeñando la parte cognitiva y social de la escritura (...) y las condiciones de contexto que requiere para la disciplina” (p. 16).

Teniendo en cuenta lo anterior, y a juicio de Torres (2004), las prácticas de escritura en universidades latinoamericanas requieren de investigaciones desde una concepción sociocultural, puesto que este acercamiento posibilita una mayor interpretación de las dificultades de escritura durante la formación profesional de los jóvenes, ya que "la confianza en que sí es posible escribir se ve reforzada por las propuestas (...) cognitivistas (...) a través de (...) estrategias o instrucciones y desde concepciones socioculturales (...) que promueven el análisis, la discusión y la confrontación” (p. 97).

Como parte de las experiencias y publicaciones latinoamericanas sobre la enseñanza de y con la lectura y la escritura en la universidad, Carlino (2013) considera que "escribir y 


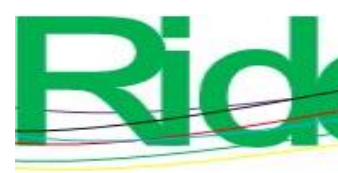

Revista Iberoamericana para la Investigación y el Desarrollo Educativo ISSN $2007-7467$

leer en cierto contexto, y reflexionar sobre ello, sirve como experiencia. Mas no forja 'la' capacidad de hacerlo de una vez para siempre y en solitario" (p. 360).

En esta tesitura, García (2017) espera que, aunque la institución no los forme ni les obligue a producir investigaciones en el área, las nuevas generaciones de docentes universitarios entiendan la importancia de contribuir interinstitucionalmente para dejar de hacer una ciencia “casera” al servicio de trámites (p. 176).

En cuanto a los programas y centros de escritura, Molina y López (2020) revelan que, sin abandonar los cursos iniciales, "en Latinoamérica, al estar la gran mayoría de programas en una fase inicial, se esté considerando a los centros y programas de escritura como una estrategia que puede contribuir al mejoramiento de las prácticas de escritura (y lectura)" (p. 112).

Ahora bien, en el caso de la FACE, existen dos programas educativos, la LEM y la Lic. en Ciencias de la Educación (LCE), cuyas comunidades de estudiantes presentan limitaciones en sus habilidades escritoras: esa fue la percepción de partida. Por ello, se revisaron los espacios donde había indicios de existir una cultura escrita a fin de indagar los siguientes cuestionamientos: ¿cuántas horas a la semana escribían los estudiantes?, ¿cuáles eran los temas de escritura?, ¿cuáles eran las dificultades a las que se enfrentaban al escribir un texto?, ¿escribían en solitario o en compañía?, ¿cuáles eran las estrategias recurridas al escribir?, ¿cuál era el lugar usado para la escritura?

A partir de estas interrogantes de investigación fue planteada la siguiente hipótesis: si los estudiantes de la LEM de la UAS disponen de espacios y cursos orientados a la práctica de la cultura escrita durante su formación profesional mayor será su desempeño académico en la universidad. Con ello, uno de los objetivos específicos de este trabajo tiene relación directa con la tarea de identificar y analizar las prácticas de escritura para el aprendizaje de la disciplina profesional de jóvenes en educación. 


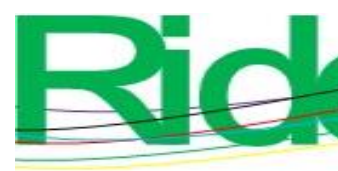

Revista Iberoamericana para la Investigación y el Desarrollo Educativo ISSN $2007-7467$

\section{Metodología}

De corte cualitativo, la metodología se apoya en la entrevista semiestructurada con enfoque sociocultural, puesto que pondera los procesos sociales del aprendizaje sobre los individuales. Para ello, se entrevistaron a 21 sujetos de un grupo de octavo semestre, opción escolarizada, quienes ya habían cursado asignaturas en torno a la enseñanza, seminarios de investigación y prácticas profesionales. Las entrevistas, de 12 preguntas, se realizaron en el mismo día y por separado. Respecto a la sistematización de los datos, se dio por agrupación de respuestas, de acuerdo con el orden de las preguntas, y para la categorización analítica, mediante la saturación de la información.

El grupo de estudio estuvo conformado por dos hombres y 19 mujeres, entre 20 y 28 años de edad, cuyas obligaciones extraescolares tenían que ver con deberes, en su mayoría, laborales, matrimoniales y paternales. La muestra fue tomada de un grupo completo, sin sesgo de género, al existir una predominancia natural de mujeres en cada uno de los grupos. De acuerdo con los datos proporcionados, los estudiantes debían marcharse al trabajo, a cuidar de los hijos o a atender a los padres al término de las clases.

Las conversaciones y las expresiones emitidas por el alumnado que rondaba por estos lugares, digamos, dispuestos para la práctica literaria, también se registraron, porque tenían que ver con dificultades para instalarse a leer o escribir. Estudiantes de diversos grados fueron observados trabajando en el Centro de Informática de la universidad, único espacio preparado con mesas, computadoras y proyector; de cualquier forma, el resto de los espacios también fue observado y registrado.

En una segunda etapa, se aplicaron entrevistas a cuatro de los profesores asignados al grupo estudiado. La entrevista semiestructurada debía mostrar en qué medida los estudiantes estaban produciendo textos académicos y cuáles eran sus prácticas escriturales. En una tercera etapa, se indagaron las actividades de educación literaria en la comunidad escolar.

\section{Resultados}

Los resultados que se muestran enseguida se derivan de las entrevistas realizadas, cuyas respuestas fueron analizadas y categorizadas por agrupación y saturación de la información, a fin de conocer cuántas horas a la semana escribían los estudiantes, cuáles serían los temas más recurridos en sus escritos, qué dificultades estaban enfrentando en la 


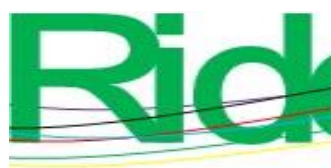

Revista Iberoamericana para la Investigación y el Desarrollo Educativo ISSN $2007-7467$

redacción, si se escribía en solitario o en compañía, cuáles estrategias se usaban al escribir y en dónde se escribía.

\section{Las horas y los temas de la escritura}

Respecto a cuántas horas escriben por semana los estudiantes, ocho horas de escritura figura por encima del rango de 15 horas, promedio semanal que se ubica en el segundo lugar de menciones, y, en tercer lugar, de dos a tres horas por semana. En esta situación, hay una disposición de tiempo insuficiente para la escritura, a pesar de tener en claro la necesidad de programar más horas para esta práctica.

Tabla 1. Las horas que los estudiantes escriben por semana

\begin{tabular}{|l|l|l|}
\hline \multicolumn{2}{|l|}{ 1) ¿Cuántas horas a la semana escribes? } \\
\hline Ocho horas, dependiendo de la inspiración & 5 estudiantes & $23.80 \%$ \\
\hline Dos o tres horas & 3 estudiantes & $14.28 \%$ \\
\hline Cero horas & 1 estudiantes & $4.76 \%$ \\
\hline 10 horas más o menos & 2 estudiantes & $9.52 \%$ \\
\hline 15 horas, dependiendo si tengo muchas tareas & 4 estudiantes & $19.04 \%$ \\
\hline 12 horas & 1 estudiante & $4.76 \%$ \\
\hline Seis horas & 2 estudiantes & $9.52 \%$ \\
\hline Cuatro horas & 1 estudiante & $4.76 \%$ \\
\hline Cinco horas & 1 estudiante & $4.76 \%$ \\
\hline No mucho & 1 estudiante & $4.76 \%$ \\
\hline
\end{tabular}

Fuente: Elaboración propia

Así pues, la mayoría tiene poco tiempo para escribir; y cuando lo tienen, en ocasiones, depende de la inspiración del momento para hacerlo o no. Aunque realmente no hay mucho qué escribir en la FACE, apenas nada, dos, tres, cuatro, cinco, seis y hasta ocho horas, porque solo al término de los cursos los profesores solicitan ensayos, informes y proyectos de servicio social, así que con ese tiempo alcanza para realizar las entregas exitosamente.

Invariablemente, se observan estas actuaciones cuando los jóvenes se quejan de las tareas escolares, debido a la enorme cantidad de textos académicos que tienen que reportar a los docentes. De acuerdo con ello, los sujetos estudiados escriben y leen menos de lo que 


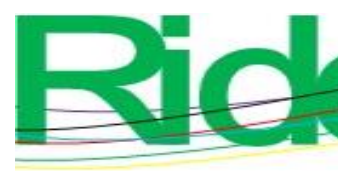

Revista Iberoamericana para la Investigación y el Desarrollo Educativo ISSN $2007-7467$

hablan. En su lugar, los requerimientos a nivel superior exigen leer mucho más para escribir que el hablar para compartir impresiones.

Sin duda no hay una manera correcta de hacerlo y, aunque se pueden cometer errores, con la práctica cada vez se adquiere la seguridad de que se escribe mejor: "Si el hecho de escribir nos angustia tanto, generalmente es porque creemos que hay una manera correcta de hacerlo, y que podríamos cometer errores (...). Nos vamos haciendo más seguros, sabemos cómo se hace, pero seguimos exigiéndonos cada vez más” (Meek, 2008, p. 43).

En la búsqueda de tiempo para desarrollar textos surgen expresiones que dicen contar con más tiempo para escribir que para leer, aunque se requiere leer por lo menos 11 horas a la semana. A decir de los estudiantes, en este centro escolar hay una exigencia por la lectura y, en la mayoría de las asignaturas, se pide un ensayo al final del curso para evaluar los aprendizajes.

Así, de siete estudiantes que dicen disponer de tiempo suficiente para la redacción, están los que afirman escribir por 15 horas a la semana, mientras que otros dos cuentan que todos los días escriben mucho, algo así como 10 horas. Incluso hay quienes pasan 12 horas en este ejercicio, pero solo cuando tienen muchas tareas; si no, no lo hacen.

En realidad, durante el proceso, los alumnos aprenden estructuras textuales. No obstante, cuando afirman que leen poco y escriben más, lo hacen sobre la experiencia de tomar nota durante las horas de clase. Por ello, resulta dudoso que los estudiantes estén produciendo escritos académicos con argumentos cuando la mayoría lee muy poco para sustentarlos. Aunque para la elaboración de textos de fin de curso lo que aseguran en sus respuestas sí aplica.

En cuanto a los temas que se escriben, los mismos aprendices estiman escribir narrativas, cuentos, de amores imposibles, poesías y canciones, sobre las experiencias personales de la vida y tareas y apuntes de clase. Por tanto, la composición literaria se repite en las expresiones dadas en los sujetos examinados, ya que, al interrogarlos para conocer la temática de escritos, las respuestas muestran cómo los discursos literarios están por arriba de los académicos y experiencias personales. 


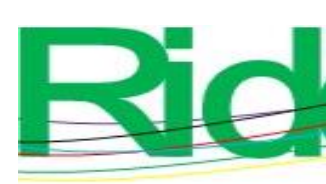

Revista Iberoamericana para la Investigación y el Desarrollo Educativo ISSN 2007 - 7467

Tabla 2. De los temas que escriben los estudiantes

\begin{tabular}{|l|l|l|}
\hline 2) ¿Cuáles son los temas de los que escribes? \\
\hline Cuentos, de amores imposibles, poesía y canciones. & 16 estudiantes & $76.19 \%$ \\
\hline Experiencias personales de la vida. & 2 estudiantes & $9.52 \%$ \\
\hline Tareas y apuntes de clase. & 3 estudiantes & $14.28 \%$ \\
\hline
\end{tabular}

Fuente: Elaboración propia

Como se puede observar, 16 jóvenes mencionan desarrollar más textos literarios, y a la narrativa la posicionan como base de sus apuntes ya que les permite realizar las tareas académicas con entretenimiento. Estos informantes cursan estudios de español y tienen conocimiento de los géneros, de la lengua y de literatura, lo cual les facilita la creación poética y narrativa, no solo en el espacio escolar, sino incluso fuera de él.

Desde este punto de análisis, al escribir discursos literarios, ellos eligen la narrativa, mientras otros prefieren escribir canciones poéticas, y hay quienes eligen crear poemas. En estas prácticas los estudiantes no intercambian referentes literarios distintos a los que se trabajan en las clases de lengua y literatura. "La escritura es una actividad, ya sea realizada en colaboración o individualmente. 'Social' no es un sinónimo de colectivo, sino que se refiere a las relaciones que existen dentro de determinada formación social" (Kalman, 2003, p. 206).

En este escenario, las prácticas escritas existentes en determinada formación social sirven como un instrumento pedagógico con el cual los estudiantes se apropian de diferentes estructuras.

También los resultados muestran cómo los educandos elaboran discursos académicos: cuando uno de ellos escribe informes de la observación docente, otro solo escribe lo que se ocupa en el aula y uno más sobre reportes de lectura previos a la clase; pero ninguno refiere poner sus textos en revisiones grupales.

Se escribe para ser revisor de su propio texto, ya que en la escuela el revisor es el maestro, pero el estudiante sabe que solo es el encargado de ponerle bien o mal y le entrega su tarea para que el maestro cumpla con su papel de revisor (Ferreiro, 2011, p. 211). 


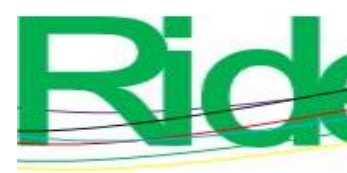

Revista Iberoamericana para la Investigación y el Desarrollo Educativo ISSN $2007-7467$

De acuerdo con esta cita, la escritura representa un objeto de enseñanza para que se constituya como un objeto de aprendizaje. No obstante, en la institución examinada, los alumnos no se involucran en la revisión de sus propios textos: dan por sentado que la voz cantante proviene del docente.

En este caso, las prácticas escriturales concentran experiencias de la vida para los jóvenes, cuando a diario se entregan informes del acontecer educativo, cuyos hechos les sirven para crear narrativas. Los participantes manifiestan redactar textos distintos a los exigidos en la escuela. Por tanto, la escritura de discursos narrativos es como escribir sobre las experiencias, aunque el escrito no sea tomado en cuenta para su evaluación. Lerner (2011) lo pone en los siguientes términos:

Lo posible es hacer el esfuerzo de conciliar las necesidades inherentes a la institución escolar con el propósito educativo de formar lectores y escritores, para generar condiciones didácticas que permitan poner en escena una versión escolar de la lectura y escritura más próxima a la versión social de estas prácticas (p. 32).

Finalmente, las circunstancias que generan documentos de experiencias de vida mueven a los estudiantes a generar una versión distinta de las prácticas de escritura impuestas en la escuela. Y en este ejercicio se aventuran a componer textos narrativos considerándolos textos íntimos. Lo interesante sería conocer las particularidades de los textos con los cuales se sienten libres para escribir.

\section{Las dificultades y las estrategias al escribir}

En cuanto a las dificultades que enfrentan los estudiantes al escribir un texto, destaca la cuestión gramatical como principal obstáculo para avanzar en la redacción académica, las distracciones y el tener que ordenar las ideas con anticipación, así como el tomar una estructura y hacer un esquema.

Ante todo, está el escribir sin faltas de ortografía y sin errores en la puntuación y acentuación, porque es una situación que dificulta la composición a poco más de la mitad de los informantes. Están más pendientes, dicen ocuparse más de atender las reglas gramaticales, puntuación y acentuación, en lugar de argumentar, insertar la voz, contextualizar, discutir una temática y llegar a una conclusión. Prueba de ello está en que la mitad de los entrevistados 


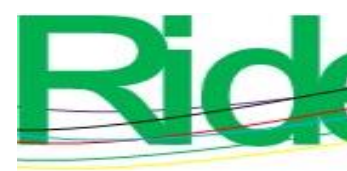

Revista Iberoamericana para la Investigación y el Desarrollo Educativo ISSN $2007-7467$

Si los estudiantes desconocen las directrices del escrito que espera el profesor ni cómo será evaluado, si coincide con la tarea esperada o está equivocado, cuando les es solicitado un texto, se buscan las referencias bibliográficas un par de días antes de la fecha de entrega y pasan de largo el tiempo de planear.

Para un escolar del grupo investigado parece muy fácil distraerse al escribir si hace otras cosas al mismo tiempo. En esta situación, le resulta complicado desarrollar un escrito escuchando ruidos, voces y pláticas de otros estudiantes. Dentro de los distractores más fuertes están las redes sociales, ya que les roba tiempo estar texteando. Con ello, poner en práctica los recursos cognitivos, lingüísticos y estratégicos, para estos escolares, significa lidiar con distractores que hacen perder la motivación para desarrollar discursos escritos. Como lo dice Lerner (2011):

El desafío está en promover el descubrimiento y la utilización de la escritura como instrumento de reflexión sobre el propio pensamiento, como recurso insustituible para organizar y reorganizar el propio conocimiento, en lugar de mantener a los alumnos en la creencia de que la escritura es solo un medio para reproducir pasivamente o para resumir el pensamiento de otros (p. 42).

Están los estudiantes con dificultades y múltiples distractores a la hora de escribir, pero también están los que toman la escritura como un instrumento de reflexión, y hacen frente a los distractores, asumen los riesgos de verse sustraídos del hilo de la escritura, y, al final, no se dejan seducir por la idea de copiar y pegar los planteamientos teóricos ajenos, sino que dan paso a su propia voz como autor.

Al momento de desarrollar textos académicos, poéticos, narrativos, apuntes o personales se tiene todo a favor. Por tanto, existe una porción de chicos, mínima, a los que se les facilita redactar sin ninguna traba, porque son individuos hábiles en la escritura.

En cuanto a las estrategias recurridas al escribir, en este estudio se muestran el trazar una ruta, hacer un borrador, buscar palabras de entrada, contextualizar, elaborar mapas conceptuales, recuperar ideas, buscar sinónimos, escuchar música, leer mucho del tema antes de escribir, releer en voz alta mientras se escribe y ninguna: son respuestas que indican en qué medida ellos se están moviendo para construir discursos. 


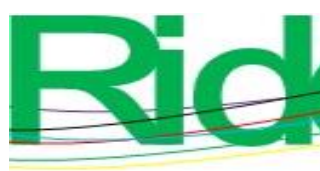

Revista Iberoamericana para la Investigación y el Desarrollo Educativo ISSN 2007 - 7467

Tabla 4. Las estrategias que usan los estudiantes para escribir

\begin{tabular}{|l|l|l|}
\hline 4) ¿Cuáles son las estrategias que usas cuando escribes? \\
\hline Escuchar música mientras escribo. & 1 estudiante & $4.76 \%$ \\
\hline Ninguna. & 6 estudiantes & $28.56 \%$ \\
\hline $\begin{array}{l}\text { Trazar una ruta; hacer un borrador; buscar palabras de } \\
\text { entrada; contextualizar; mapas conceptuales; recuperar } \\
\text { ideas; buscar sinónimos. }\end{array}$ & 11 estudiantes & $52.36 \%$ \\
\hline $\begin{array}{l}\text { Leer mucho del tema antes de escribir. } \\
\text { Releer en voz alta mientras escribo. }\end{array}$ & 1 estudiante & $4.76 \%$ \\
\hline
\end{tabular}

Fuente: Elaboración propia

Hay alumnos que trazan una ruta al momento de escribir y quienes, antes de comenzar un escrito, hacen, construyen un borrador o mapas conceptuales, además de recuperar las ideas principales, a fin de formar un índice que les permita contextualizar los fundamentos teóricos. "Al escribir se implica relacionar, jerarquizar y estructurar el caos del pensamiento primario" (Carlino, 2005, p. 18).

En este grupo educativo se ensaya la escritura al trazar una ruta para reflexionar sobre contenidos programáticos, jerarquizar ideas y estructurar el pensamiento en párrafos. Y si a este proceso se añade leer mucho del tema y releer en voz alta al escribir, los educandos consiguen un texto sustentado, si antes se empapan del tema. Por lo regular, estas prácticas se utilizan como apoyo durante el proceso de escritura y les permiten irse de corrido sin parar hasta concluir el texto.

La práctica es fundamental en la antelación del discurso escrito. En torno a la lectura y relectura, están los jóvenes que manifiestan leer y releer antes y durante la escritura porque con esto obtienen información valiosa del tema que deben escribir. Aunque para idear un escrito no basta con trazar una ruta, leer y releer, también se requiere, a juicio de Castro y Sánchez (2013): 


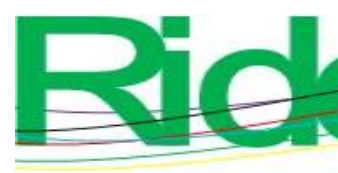

Revista Iberoamericana para la Investigación y el Desarrollo Educativo ISSN $2007-7467$

Discutir una propuesta, plantear un problema, defender una tesis, evaluar un marco teórico o metodológico o construir una conclusión requieren del hablante o del escritor habilidades que van más allá del simple dominio de las convenciones lingüísticas. Estas prácticas académicas, entre muchas otras, exigen a los estudiantes saber leer textos especializados (p. 504).

Asimismo, se requiere asumir posturas y construir opiniones objetivas y sustentadas en evidencias pertinentes al campo disciplinar de la adhesión o la confrontación de las distintas opiniones o puntos de vista vertidos por otros en la práctica de escritura. "No es una tarea fácil, al requerirse de un aprendizaje sistemático que apunte al discurso especializado" (Castro y Sánchez, 2013, p. 504).

Algunas respuestas evidencian la insuficiencia de estrategias para escribir, ya que una tercera parte del grupo estudiado desconoce tácticas de escritura. Aún quedan por examinar los textos que están produciendo para conocer en qué medida usan o no estrategias textuales narrativas para elaborar los discursos escritos.

\section{En solitario, en compañía y el lugar donde se escribe}

Respecto a si se escribe en solitario o en compañía, es de resaltar las respuestas que indican que sucede una combinación de ambos modos, en soledad y en compañía, porque todo depende de las circunstancias. Por otra parte, al buscar sobre el lugar donde usualmente se está redactando, los participantes se decantan por un lugar sin ruido y con muebles específicos y atmósfera propicia para la lectura y escritura. Aunque, en menor medida, también funcionan como espacios donde se practica la escritura la recamara, la cafetería y la casa.

En realidad, una tercera parte de los informantes declaran que es mejor trabajar los textos en solitario porque así pueden concentrarse y aprender más. Por lo regular, según lo manifestado, redactar en solitario les permite estar más seguros de sus propias habilidades de escritura, una vez que se han acostumbrado a retirarse del mundo para producir discursos, porque no siempre la escuela les permite ese tiempo de reescritura en compañía. 


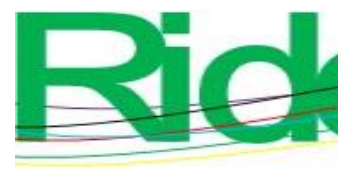

Revista Iberoamericana para la Investigación y el Desarrollo Educativo ISSN 2007 - 7467

Tabla 5. La escritura en solitario o en compañía de otros estudiantes

\begin{tabular}{|l|l|l|}
\hline 5$)$ ¿Escribes en solitario o en compañía? \\
\hline En solitario & 7 estudiantes & $33.32 \%$ \\
\hline En solitario y en compañía & 14 estudiantes & $66.64 \%$ \\
\hline
\end{tabular}

Fuente: Elaboración propia

Si bien la mayoría de los informantes escribe tanto en solitario como en compañía, de acuerdo con sus expresiones, encuentran que redactar en solitario solo sirve para obtener información de forma rápida, a diferencia de hacerlo en compañía, modo en que es posible observar los errores de los otros para evitarlos en el propio texto. Por tanto, la escritura en solitario, en compañía o de ambas formas no les resulta igual en términos de aprendizaje de los contenidos.

En este escenario, surge la inquietud de sopesar la importancia de escribir en solitario o en compañía, donde resulta de suma utilidad que los estudiantes participen en la revisión de los textos de sus compañeros de manera obligada, para construir el sentido y obtener el placer de entender más y mejor con la escritura. Andrade y García (2015) lo exponen así:

Para el docente porque con la coevaluación aumentan las probabilidades de conseguir detalles de corrección que una sola persona, indudablemente, no podría percibir, mientras que para el estudiante resulta fructífero porque las inconsistencias detectadas en los textos ajenos le pudieran servir para evitarlas en los suyos (p. 463).

Teniendo en cuenta lo anterior, cobra sentido la demanda de contar con un espacio propio donde puedan sentirse cómodos los escolares para escribir sin prisas ni sobresaltos por el calor, los ruidos que aumentan en lugares reducidos y de poco mobiliario, sobre todo cuando ellos mismos piden directamente un lugar que tenga ventanas grandes con vista a los árboles, con escritorios grandes y sillas cómodas, que esté fresco y limpio, con mucha luz y que sea tranquilo, cómodo, luminoso y sin mucho ruido. 


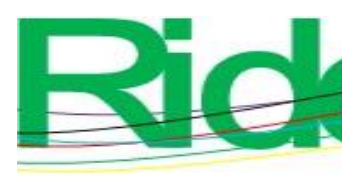

Revista Iberoamericana para la Investigación y el Desarrollo Educativo ISSN 2007 - 7467

Tabla 6. El lugar donde usualmente escriben los estudiantes

6) ¿Cuál es el lugar donde usualmente escribes?

\begin{tabular}{|l|l|l|}
\hline Indistinto & 3 estudiantes & $14.28 \%$ \\
\hline En mi cuarto, en casa & 5 estudiantes & $23.8 \%$ \\
\hline En la biblioteca de la escuela & 1 estudiante & $4.76 \%$ \\
\hline $\begin{array}{l}\text { Donde esté una ventana con vista al paisaje con árboles. } \\
\text { Donde haya un escritorio, en un lugar que esté fresco y } \\
\text { limpio, que tenga una silla cómoda. En una mesa grande, } \\
\text { en un cuarto pequeño con aire acondicionado. En un lugar } \\
\text { amplio con luz. En una superficie fija y que sea un lugar } \\
\text { tranquilo. Cómodo y luminoso y sin mucho ruido. }\end{array}$ & $57.12 \%$ \\
\hline
\end{tabular}

\section{Fuente: Elaboración propia}

Los centros educativos están obligados a favorecer un cambio en los agentes de distribución, circulación y acceso a los materiales de lectura, con el propósito de que en el ámbito de la formación profesional se promueva una cultura escrita que rompa con la cultura extraescolar de leer y escribir en cualquier lugar menos en la escuela. "Es probable que el mayor número de centros de escritura versus el número de programas, tenga relación a que se proponen como estrategia de trabajo individual frente al trabajo grupal de los cursos" (Molina y López, 2020, p. 114).

Una cuarta parte del grupo de estudio desarrolla los discursos escritos en casa porque les resulta un lugar donde se pueden concentrar fácilmente durante la redacción. Contrario a esto, observamos que en la escuela los espacios para la escritura no encajan con el concepto idóneo para esta práctica que los estudiantes tienen, cuya descripción incluye amplitud, iluminación, sin ruido, fresco y con una mesa grande. Al parecer todo esto lo tienen en casa. Esto tiene que ver con estudiantes que reciben muy poco apoyo de los docentes sobre las tareas escriturales cuando, por lo regular, no se realiza una retroalimentación de lo evaluado en el escrito.

Por tanto, se está escribiendo en la escuela y en la casa, adaptándose al tiempo y a las circunstancias, a fin de mantenerse en los aprendizajes. Para los informantes, lo más importante es asumirse como lectores y escritores de a pie y de a diario. Aunque sería muy gratificante la interacción con docentes como tutores que están al pendiente del proceso de construcción y aprendizaje de sus estudiantes. Carlino (2016) menciona: 


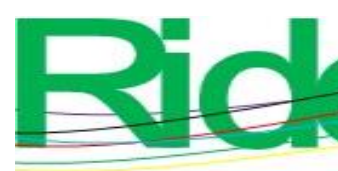

Revista Iberoamericana para la
Investigación y el Desarrollo Educativo
ISSN $2007-7467$

Confirmamos que son las actividades que se realizan durante las clases y entrelazan el trabajo sobre la lectura y la escritura con los temas propios de las materias. En menor medida aún, algunos docentes expresan que promueven la interacción entre profesor y alumnos o entre pares, a través de la discusión sobre lo leído o lo escrito (p. 3).

Este resultado muestra que hay una minoría de escolares elaborando escritos en sus centros educativos porque pueden escribir y consultar libros como referentes para sustentar el discurso, aunque "la interacción social, por sí misma, no genera conocimientos ni promueve, necesariamente, el desarrollo cognoscitivo (...). De la misma manera, se reconoce que esto no ocurre en cualquier contexto o ambiente" (Dembo y Guevara, 2001, p. 145).

Por último, la mayoría de ellos, al escribir, se ajustan a las condiciones del momento, ya sea en casa, en la biblioteca o en cualquier lugar distinto a la escuela, en solitario, en compañía o de ambas formas, porque es lo que se tiene, siempre con el objetivo de aprender de lo que se escribe en la formación universitaria.

\section{Discusión}

Este estudio resalta la manera en que influye o afecta el entorno al proceso de escritura de los jóvenes universitarios. En este caso, en el entorno de formación profesional, los estudiantes manifiestan dificultades y necesidades más recurrentes al momento de escribir en la escuela. Estas se enlistan en el siguiente orden: la insuficiencia de horas dedicadas a escribir; la dificultad para escribir atendiendo la cuestión gramatical; el dominio de la escritura del discurso literario; el trazar una ruta como estrategia de escritura, y la preferencia de escribir en solitario y en un lugar con todo lo necesario para la lectura y escritura por tiempo indefinido.

En este ambiente aparece una urgencia de escribir arriba de ocho horas a la semana para superar las dificultades gramaticales en los discursos escritos. Ante todo, se desbordan las participaciones que demandan un espacio escolar (amplio, luminoso, refrigerado, con mesas grandes, ventanas amplias con vista a los árboles) del que se carece en la escuela y en casa y que, a fin de salir adelante en los estudios superiores, para una minoría, una habitación en casa se convierte en la tabla de salvación para fijar los aprendizajes a través de la lectura y escritura en solitario o en compañía. 


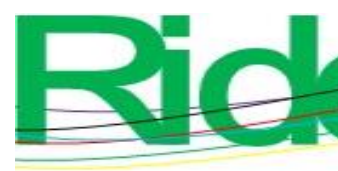

Revista Iberoamericana para la Investigación y el Desarrollo Educativo ISSN $2007-7467$

En la línea de cultura escrita existen diferentes planteamientos que sugieren el acceso a la cultura escrita a través de la interacción con otros lectores, otros escritores y sujetos que viven el día entre leer y escribir (mucho), el contexto en que se produce y la situación de quien escribe y deja de reproducir pasivamente el pensamiento de otros. La enseñanza y aprendizaje de la escritura desde los enfoques, cognitivo y lingüístico y atendiendo la cuestión gramatical y a través de la lectura desde lo individual. La escritura como una práctica sociocultural para escribir en compañía y aprender de los errores de los otros. La enseñanza para el aprendizaje de la escritura en el primer o último grado como antelación a los discursos escritos que habrán de desarrollarse en otras asignaturas. La escritura en contexto y grupal porque en todas las asignaturas se elaboran textos académicos con sus particularidades. La escritura dada en centros de escritura, de manera colaborativa, tomando como aprendizaje lo escrito por los otros para escribir y reescribir los textos. A juicio de Carlino (2017):

Si la lectura y la escritura convierten al conocimiento en un objeto para pensar y sobre el que se puede discutir con otros, ¿tiene sentido confinarlas solo a unos pocos cursos, aquellos que pretenden enseñarles esas prácticas específicas, o a tutorías externas a las materias? (p. 30).

Dentro de las fortalezas del estudio, cuenta con informantes inscritos en una licenciatura dirigida a la enseñanza del español, no obstante el programa educativo incluye otras áreas del conocimiento (seis asignaturas por semestre): psicología, sociología, tecnología, práctica docente, lengua extranjera y lengua materna, donde para evaluar los aprendizajes, además de discursos literarios, se solicitan ensayos, controles de lectura, resúmenes, informes de observación de la práctica docente y de servicio social. Con todo, para completar este estudio, habría que indagar sobre las características de los discursos literarios para identificar las particularidades del entorno donde se gestan. 


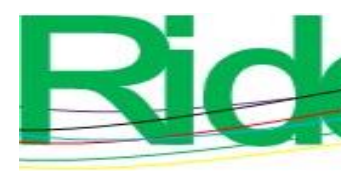

Revista Iberoamericana para la Investigación y el Desarrollo Educativo ISSN $2007-7467$

\section{Conclusiones}

La escritura académica en la universidad requiere de la escritura y reescritura en compañía: en la sesión de clase, en las asignaturas, en la escuela, en grupos presenciales y virtuales, a fin de intercambiar opiniones, estrategias y estructuras discursivas en los espacios propios de formación disciplinar, ya que las prácticas individuales conducen a composiciones textuales sobre una base narrativa que solo tiene lugar en su contexto real de desarrollo.

A partir de esta investigación queda confirmado que los universitarios, al disponer de lugares, ya sea presenciales, virtuales o mixtos, y cursos orientados a la práctica de una cultura escrita, aumentan el desempeño académico de su formación profesional, lo que significa escribir en el contexto mismo en que desarrollan y tienen lugar las diferentes prácticas de cultura escrita en la universidad.

Esto apunta a plantear y diseñar espacios escolares que favorezcan las prácticas de una cultura escrita. Prácticas que conllevan a la investigación educativa y al desarrollo de actividades orientadas a la educación literaria. Y sobre todo a crear espacios para la escritura en compañía, sin menospreciar el carácter cognitivo que sustenta a la escritura académica. También pudiéramos incluir los centros de escritura: aunque sean insuficientes para resolver el problema, significan un fuerte apoyo para las tutorías de los docentes, pues allí los estudiantes reciben acompañamiento en el proceso de escritura de las tareas escolares, de investigación educativa y de creación literaria.

Cierto, la escritura en compañía como tema de investigación requiere de nuevas líneas de investigación que retomen el contexto actual de la pandemia por el SARS-CoV-2, el confinamiento social y la incorporación masiva de las tecnologías en los procesos de formación universitaria. De aquí que las formas de relación e interacción entre los sujetos escritores tendrán que adecuarse a esta nueva realidad social, donde la escritura en compañía será el principal aliciente para el autoaprendizaje. 


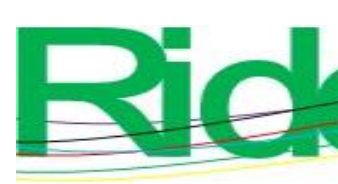

Revista Iberoamericana para la
Investigación y el Desarrollo Educativo
ISSN $2007-7467$

\section{Futuras Líneas de Investigación}

Así, con la revisión de literatura y los hallazgos presentados en esta investigación, queda demostrada la insuficiencia de investigación sobre las prácticas de cultura escrita en los contextos de formación superior en México. Por lo que, la escritura y reescritura de textos académicos adquiere relevancia, como tema de investigación, al plantearse las siguientes preguntas: ¿Qué lugar ocupa la escritura académica en los distintos programas de licenciatura? ¿Qué están escribiendo los jóvenes durante su formación profesional? ¿De qué recursos se vale el docente para que el estudiante aprenda a escribir y leer textos propios de la disciplina de estudio profesional? ¿Cómo resuelve el estudiante sus deficiencias de lectura y escritura en la universidad? ¿Cuáles son las estrategias de lectura y escritura que promueven los docentes en las distintas carreras profesionales? ¿Cuáles son, desde la perspectiva del docente, las principales deficiencias de lectura y escritura que presentan los jóvenes en la universidad?

\section{Referencias}

Andrade, E. y García, M. (2015). Investigaciones sobre escritura académica realizadas por la comunidad científica de la Universidad de Los Andes (2009-2013): Alcances y Limitaciones. Educere, mayo-agosto 19(63),455-468. Mérida, Venezuela: Universidad de los Andes. Recuperado de https://www.redalyc.org/pdf/356/35643049010.pdf

Carlino, P. (2004). El proceso de escritura académica: cuatro dificultades de la enseñanza universitaria. Educere, 026(8), 321-327. Recuperado de https://www.redalyc.org/pdf/356/35602605.pdf

Carlino, P. (2005). Escribir, leer, y aprender en la universidad. Una introducción a la alfabetización académica. Buenos Aires, Argentina: Fondo de Cultura Económica.

Carlino, P. (2013). Alfabetización académica. Diez años después. Revista Mexicana de Investigación Educativa, 18(57), 355-381 Recuperado de https://www.redalyc.org/pdf/140/14025774003.pdf.

Carlino, P. (2016). Lectura y escritura al servicio del aprendizaje de diversas materias: estudios del Giceolem en formación docente inicial. Ponencia presentada en las Jornadas Internacionales de Escritura y Alfabetización. La Plata, del 17 al 19 de 


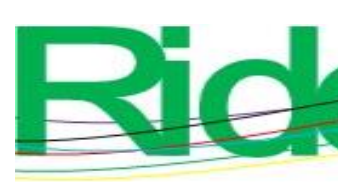

Revista Iberoamericana para la
Investigación y el Desarrollo Educativo
ISSN $2007-7467$

noviembre

de

2016.

Recuperado

de

https://www.aacademica.org/paula.carlino/229.pdf.

Carlino, P. (2017). Dos variantes de la alfabetización académica cuando se entrelazan la lectura y la escritura en las materias. Signo y Pensamiento, 36(71), 16-32. Recuperado de http://www.redalyc.org/articulo.oa?id=86054913001.

Carrasco, A., Encinas, M., Castro, M. y López, G. (2013). Lectura y escritura académica en la educación media superior y superior. Revista Mexicana de Investigación Educativa, 18(57), 349-354. Recuperado de https://www.redalyc.org/pdf/140/14025774002.pdf.

Castro, M. y Sánchez, M. (2013). La expresión de opinión en textos académicos escritos por estudiantes universitarios. Revista Mexicana de Investigación Educativa, 18(57), 483-506. Recuperado de https://www.redalyc.org/pdf/140/14025774008.pdf.

Dembo, M. y Guevara, M. (2001). Desarrollo psicológico, aprendizaje y enseñanza: una comparación entre el enfoque socio-cultural y el análisis conductual. Revista Latinoamericana de Psicología, 33(2), 141-147. Recuperado de https://www.redalyc.org/pdf/805/80533203.pdf.

Ferreiro, E. (2011). Cultura escrita y educación. México: Fondo de Cultura Económica.

García, M. (2004). Aproximación al estudio de las representaciones de los docentes universitarios sobre el ensayo escolar. Revista de Teoría y Didáctica de las Ciencias Sociales, (9), 9-33. Recuperado de https://media.utp.edu.co/referenciasbibliograficas/uploads/referencias/articulo/551-aproximacion-al-estudio-de-lasrepresentaciones-de-los-docentes-universitarios-sobre-el-ensayo-espdf-70xmXarticulo.pdf.

García, M. (2017). Inventario de artículos de investigación sobre escritura y lectura académica producidos por miembros de la Universidad de Los Andes (Venezuela). Revista Acción Pedagógica, (26), 176-184. Recuperado de https://dialnet.unirioja.es/servlet/articulo?codigo $=6344983$.

Kalman, J. (2003). Escribir en la plaza. México: Fondo de Cultura Económica.

Kaufman, A. (2005). Cómo enseñar, corregir y evaluar la ortografía de nuestros alumnos... y no morir en el intento. Lectura y Vida: Revista Latinoamericana de Lectura, 26(3), 6-20. Recuperado de https://dialnet.unirioja.es/servlet/articulo?codigo=1299778. 
Lerner, D. (2011). Leer y escribir en la escuela: lo real, lo posible y lo necesario. México: Fondo de Cultura Económica.

Meek, M. (2008). En torno a la cultura escrita. México: Fondo de Cultura Económica.

Molina, V. y López, K. (2020). Estado de la cuestión de los centros y programas de escritura en Latinoamérica. Revista Colombiana de Educación, 1(78), 97-119. Recuperado de http://doi.org/10.17227/rce.num78-8066.

Salazar, C. (2015). La escritura académica universitaria: diferentes perspectivas de estudio. Diálogos sobre Educación, 6(11), 1-21. Recuperado de https://www.redalyc.org/pdf/5534/553457059005.pdf.

Torres, I. (2004). Una mirada pedagógica a la escritura de un ensayo argumentativo. Revista de Estudios Sociales, (19), 97-105. Recuperado de https://journals.openedition.org/revestudsoc/24327. 


\begin{tabular}{|c|c|}
\hline Ne & $\begin{array}{l}\text { Revista lberoamericana para la } \\
\text { Investigación y el Desarrollo Educativo } \\
\text { ISSN } 2007-7467\end{array}$ \\
\hline Rol de Contribución & Autor (es) \\
\hline Conceptualización & María Luisa Guadalupe Verástica Cháidez. \\
\hline Metodología & María Luisa Guadalupe Verástica Cháidez. \\
\hline Software & Crisanto Salazar González. \\
\hline Validación & Crisanto Salazar González. \\
\hline Análisis Formal & María Luisa Guadalupe Verástica Cháidez. \\
\hline Investigación & $\begin{array}{l}\text { María Luisa Guadalupe Verástica Cháidez. } \\
\text { Crisanto Salazar González. <<igual〉> }\end{array}$ \\
\hline Recursos & María Luisa Guadalupe Verástica Cháidez. \\
\hline Curación de datos & Crisanto Salazar González. \\
\hline $\begin{array}{l}\text { Escritura - Preparación del } \\
\text { borrador original }\end{array}$ & $\begin{array}{l}\text { María Luisa Guadalupe Verástica Cháidez. } \\
\text { Crisanto Salazar González. <<igual〉> }\end{array}$ \\
\hline $\begin{array}{l}\text { Escritura - Revisión y } \\
\text { edición }\end{array}$ & María Luisa Guadalupe Verástica Cháidez. \\
\hline Visualización & Crisanto Salazar González. \\
\hline Supervisión & Crisanto Salazar González. \\
\hline Administración de Proyectos & María Luisa Guadalupe Verástica Cháidez. \\
\hline Adquisición de fondos & María Luisa Guadalupe Verástica Cháidez. \\
\hline
\end{tabular}

